\title{
LITERATURBERICHT
}

\author{
Thorsten Gromes
}

\section{Der Rückfall in den Bürgerkrieg}

Der Literaturbericht referiert die Befunde von rund 40 quantitativen Studien zur Frage, was in Nachbürgerkriegsgesellschaften einen weiteren Bürgerkrieg wahrscheinlicher macht. Ursachen erneuter Bürgerkriege lassen sich in Merkmalen des vorherigen Bürgerkrieges, der Gestalt der Nachkriegsordnung und strukturellen Eigenheiten der betroffenen Gesellschaft finden. Eine hohe Zahl von Opfern im letzten Bürgerkrieg und eine große Bedeutung des Exports natürlicher Ressourcen heben die Wahrscheinlichkeit eines neuen Bürgerkrieges. Hingegen hemmen eine starke Wirtschaft und Friedenstruppen den Rückfall in den Krieg. Widersprüchlich sind die Befunde dazu, inwieweit eine Nachkriegsordnung einen Ausgleich zwischen den Konfliktparteien schaffen soll. Auf der einen Seite gilt der Frieden nach Siegen und damit nach Durchsetzung der Anliegen einer Konfliktpartei als stabiler als nach Friedensabkommen. Auf der anderen Seite verträgt sich eine Untergruppe der Friedensabkommen, und zwar die mit Machtteilung zwischen den früheren Kriegsparteien, besonders gut mit dauerhaftem Frieden.

Schlagworte: Bürgerkriegsursachen, Friedenskonsolidierung, Friedensstrategien, Überlebensdaueranalyse

\section{Nachbürgerkriegsgesellschaften in der Konfliktfalle}

Vor einigen Jahren prägte eine Forschungsgruppe der Weltbank den Begriff »Konfliktfalle« (Collier et al. 2003) und machte mit ihm darauf aufmerksam, dass ein Bürgerkrieg seine eigenen Ursachen aufrechterhält oder sogar verstärkt, wodurch ein neuer Krieg droht (siehe auch Walter 2004). ${ }^{1}$ Studien ermittelten nach Bürgerkriegen eine Rückfallquote zwischen 17\% (Toft 2010 b: 9) und 50\% (Collier/Hoeffler 2002: 17). Welche Zahl auch zutreffen mag, in jedem Fall gilt: Nach dem Krieg ist oft vor dem Krieg. Wie allerdings Arbeiten zu Bürgerkriegsursachen zeigen, wird

1 Für konstruktive Kommentare danke ich Thorsten Bonacker, Lothar Brock, Sofia Ganter, Ute Möntnich, Hans-Joachim Spanger, Christoph Weller sowie den drei anonymen GutacherInnen. 
mit einer längeren Friedensdauer ein neuer Bürgerkrieg immer unwahrscheinlicher. $^{2}$

Die Relevanz der Forschung zu Rückfällen in den Bürgerkrieg muss man nicht lange begründen: Einsichten in die Ursachen erneut ausbrechender Bürgerkriege können dazu beitragen, einen weiteren Waffengang zu verhindern. Dieser Literaturbericht präsentiert Antworten auf die Frage, welche Einflüsse einen Rückfall in den Bürgerkrieg wahrscheinlicher machen. Für die politische Praxis kommt es vor allem darauf an, die Wirkung derjenigen Faktoren zu kennen, die sich nach einem Bürgerkrieg gestalten lassen. Der Beitrag rückt diese Faktoren in den Mittelpunkt, geht aber knapp auch auf die ein, die sich direkten Eingriffen ganz oder weitgehend entziehen. Aus zwei Gründen beschränkt er sich auf quantitative Befunde. Zum einen lassen sich die quantitativen Arbeiten im Gegensatz zu den qualitativen Studien noch relativ leicht überschauen. Zum anderen beansprucht die quantitative Forschung, im besonderen Maße über einzelne Fälle hinaus auftretende Muster zu identifizieren.

Die Basis des Literaturberichts bilden rund 40 quantitative Studien ${ }^{3}$ in der Schnittmenge der Forschungen zur Friedenskonsolidierung und zu Rückfällen in den Bürgerkrieg. ${ }^{4}$ Während sich letztere auf die Frage konzentrieren, weshalb Nachbürgerkriegsgesellschaften einen weiteren innerstaatlichen Krieg erleben, geht es Studien zur Friedenskonsolidierung nicht um die bloße Abwesenheit eines weiteren Bürgerkrieges, sondern darüber hinaus um den Bestand eines sich selbst tragenden Friedens. Die meisten Arbeiten zur Friedenskonsolidierung setzen ein Friedensabkommen oder eine andere ausgehandelte Regelung voraus. Hingegen beschränken sich die Studien zum Rückfall in den Bürgerkrieg nicht auf eine bestimmte Form des Kriegsendes. Der Literaturbericht bemüht sich, all diejenigen bis Ende 2011 publizierten Studien einzubeziehen, deren Titel einen Bezug zur Friedenskonsolidierung oder zum Rückfall in den Bürgerkrieg oder innerstaatlichen

2 Siehe Dixons Meta-Studie (2009: 717); Buhaug et al. (2008: 544); Cederman et al. (2009: 516); Cederman et al. (2011: 487); Collier/Hoeffler (2004: 576); Collier et al. (2005: 17); Collier et al. (2009: 13); Regan/Norton (2005: 330). Siehe zudem Collier (2000: 8) und Quinn et al. (2007: 186). Keine statistisch signifikante Wirkung der Friedensdauer ermittelt Gottwald (2009: 9). Laut Collier et al. (2008: 467) gibt es in den ersten Nachkriegsjahren keinen statistisch signifikanten Effekt. Folgt man der Mehrheit der Studien, nagt der Zahn der Zeit an der Wahrscheinlichkeit eines weiteren Bürgerkrieges. Das könnte daran liegen, dass mit den Jahren feindselige Einstellungen und Rachegelüste nachlassen und Waffen sowie Fertigkeiten zu deren Einsatz immer weniger verfügbar sind.

3 Hartzell/Hoddie (2007), Toft (2010 b) und Walter (2002) gehen nicht rein quantitativ vor, sondern sichern ihre Befunde mit Fallstudien ab.

4 Viele AutorInnen veröffentlichen Teile von Monographien nochmals als Aufsatz. In solchen Fällen habe ich nur eine Publikation herangezogen, so Hartzell/Hoddie (2007) und Toft (2010 b), nicht aber Hoddie/Hartzell (2003) und Toft (2010 a). 
bewaffneten Konflikt zu erkennen geben. Jedoch beansprucht er keine Vollständigkeit.

Zuerst stellt der Literaturbericht Umfang, Schwerpunkte und Vorgehen der ausgewerteten Arbeiten vor (2.). Im nächsten Schritt skizziert er die Effekte von Faktoren, die noch während des Bürgerkrieges zu beeinflussen sind (3.). Anschließend stehen Eigenheiten des Konflikts im Vordergrund, die Eingriffe von außen kaum zulassen (4.). Der Schwerpunkt liegt auf Aspekten der Nachkriegsordnung (5.); danach streift der Bericht die Befunde zu den Wirkungen ökonomischer Größen (6.). Am Schluss macht er Vorschläge für die weitere Forschung (7.).

\section{Charakteristika der quantitativen Studien}

Die meisten Studien definieren Bürgerkrieg (ähnlich) wie das Projekt Correlates of War, das unter Bürgerkrieg eine militärische Auseinandersetzung innerhalb eines international anerkannten Staates versteht, an der die Regierung teilnimmt, alle Seiten dem Feind Verluste zufügen und innerhalb eines Jahres mindestens 1000 KombattantInnen in Kämpfen oder an deren Folgen sterben (Sarkees 2010: 5-10, 15). Ein kleiner Teil der Arbeiten wertet die vom Uppsala Conflict Data Progam (UCDP) aufgelisteten bewaffneten Konflikte innerhalb von Staaten aus. ${ }^{5}$ Ein bewaffneter Konflikt liegt vor, wenn innerhalb eines Kalenderjahres 25 Menschen oder mehr durch Kämpfe ums Leben kommen. ${ }^{6}$ Der Kürze halber spricht der Bericht im Folgenden nur von »Bürgerkrieg«, auch wenn er sich auf beide vorgestellten Definitionen bezieht.

Ein unterschiedliches Verständnis von Bürgerkrieg führt zu divergierenden Ansichten darüber, wann ein solcher als erneut ausgebrochen gelten kann. Doch selbst die Arbeiten, die unter »Bürgerkrieg« das Gleiche verstehen, fassen »Rückfall in den Bürgerkrieg « unterschiedlich auf. Einige Studien berücksichtigen nur die Bürgerkriege, bei denen die gleichen Konfliktparteien um die gleichen Konfliktgegenstände wie im vorherigen Waffengang kämpfen (z.B. Fortna 2004: 276; Hartzell 2009: 356). Andere Arbeiten schauen auf jeden neuen Bürgerkrieg, auch wenn ihn andere Konfliktparteien über andere Streitfragen ausfechten (z.B. Doyle/Sambanis 2006: 135; Mason et al. 2011: 180). Ein Teil der Studien macht nicht transparent, wann ein Rückfall in den Bürgerkrieg vorliegt (z.B. Quinn et al. 2007; Toft 2010 b).

5 DeRouen et al. (2009); Kreutz (2010); Jarstad/Nilsson (2008); Meernik et al. (2010); Nilsson (2008).

6 Siehe http://www.pcr.uu.se/research/ucdp/definitions/definition_of_armed_conflict/; 8.8.2012. Vgl. demgegenüber die qualitative Definition der Arbeitsgemeinschaft Kriegsursachenforschung: http://www.sozialwiss.uni-hamburg.de/publish/Ipw/Akuf/kriege_aktuell.htm\#Def; 8.8.2012. 
Die meisten Studien rechnen mit Datensätzen, die den Zeitraum nach dem Zweiten Weltkrieg bis zu den 1990er Jahren umfassen, und kommen je nach Kriegsbegriff auf 91 (Licklider 1995) bis 124 Fälle (Doyle/Sambanis 2000).

Herrscht die logistische Regression in allgemeinen Studien über Bürgerkriegsursachen vor, dominiert in der Forschung zum Rückfall in den Bürgerkrieg die Überlebensdaueranalyse (Box-Steffensmeier/Jones 2004). Dieses Verfahren muss den betrachteten Nachkriegszeitraum nicht willkürlich auf zwei, fünf oder mehr Jahre beschränken. Stattdessen gehen die Fälle solange in die Untersuchung ein, bis ein für alle gültiger Stichtag erreicht ist.

Die allgemeine Bürgerkriegsursachenforschung hat mehr als 200 Variablen untersucht (Dixon 2009: 709); Arbeiten über den Rückfall in den Bürgerkrieg nahmen mehr als 50 Faktoren unter die Lupe, Unterformen eines Faktors nicht mitgezählt. Solche Unterformen sind etwa die vier Teilaspekte der Machtteilung (Hartzell/ Hoddie 2007: 25-36). Auch sind Interaktionsgrößen wie Machtteilung nach einem bestimmten Kriegsende (Mukherjee 2006 b: 498) nicht eingerechnet.

Machtteilung und Friedenstruppen zählen zu den am häufigsten untersuchten Größen. Der Konflikttyp, die Kriegsdauer, die Zahl der Kriegsopfer, die Art des Kriegsendes sowie Regimeform und Wirtschaftskraft ergänzen diese Reihe. Diesen Faktoren stehen einige selten analysierte Exoten gegenüber, etwa wieweit ein Friedenabkommen auf die Konfliktgegenstände eingeht (DeRouen et al. 2009: 382), ob fremde Truppen abgezogen werden (Mattes/Savun 2009: 754), die Gefechte vor allem im Gebirge stattfanden (Fortna 2008: 106), Konfliktparteien einen Separatfrieden schließen (Nilsson 2008) oder internationale Tribunale die Kriegsvergangenheit aufarbeiten (Meernik et al. 2010). Der Literaturbericht beschränkt sich auf die Faktoren, zu denen mehr als einzelne Befunde vorliegen.

Die meisten Studien zu Bürgerkriegsursachen prüfen nicht, ob ihre Variablen endogen sind (Hegre/Sambanis 2006: 513). Auch Arbeiten zum Rückfall in den Bürgerkrieg testen nur in Ausnahmefällen auf Endogenität und Selektionseffekte. So ermittelte Virginia Page Fortna (2004: 278-279), dass die Entsendung einer Friedenstruppe nach Siegen unwahrscheinlicher ist als nach Friedensabkommen.

Der Bericht legt im Folgenden dar, wie viele Arbeiten dem jeweils betrachteten Faktor einen statistisch signifikanten Effekt auf die Wahrscheinlichkeit eines weiteren Bürgerkrieges zusprechen. Dabei kann ein Faktor den Rückfall in den Bürgerkrieg hemmen oder fördern. Zudem berücksichtigt der Bericht die Studien, die keine statistisch signifikante Wirkung des untersuchten Faktors feststellen. Indem er auf die Häufigkeit der verschiedenen Befunde schaut, zeigt der Beitrag, wieweit 
sich die Forschung über eine Aussage einig zeigt. ${ }^{7}$ Ein Nachteil dieses Vorgehens besteht darin, dass es alle Studien auf eine Stufe stellt - ungeachtet der Güte der theoretischen Fundamente, Operationalisierungen, Datensätze und Auswertungsverfahren. So kommt der Studie von Anna K. Jarstad und Desirée Nilsson (2008), die nicht nur die Vereinbarung, sondern auch die Umsetzung von Machtteilung untersuchen, dasselbe Gewicht zu wie einer Arbeit, die eine Machtteilung bereits bei Verhältniswahlrecht gegeben sieht (Mukherjee 2006 b: 494). Da das Verhältniswahlrecht nicht zwingend zu einer gemeinsamen Regierung der Konfliktparteien führt, überdehnt diese Operationalisierung das Konzept der politischen Machtteilung. Der Literaturbericht könnte alternativ zum gewählten Vorgehen nur die Befunde der zuverlässigsten Studien präsentieren. Dafür müsste er jeweils genau nachweisen, was die ausgewählten Arbeiten positiv von den restlichen abhebt, und auch die eigenen theoretischen, methodischen oder politischen Vorlieben offenlegen. Zudem müsste er ein Instrumentarium entwickeln, um verschiedene Stärken und Schwächen der einzelnen Studien gegeneinander abzuwägen. Ist etwa eine Arbeit mit genauer Operationalisierung und wenigen Fällen besser als eine Studie mit einer gröberen Operationalisierung, aber deutlich umfangreicheren Datenbasis? Die höherwertigen Arbeiten von den anderen abzugrenzen würde einen so großen Begründungsaufwand verlangen, dass sich der Literaturbericht auf ein Teilsegment der Forschung zum Rückfall in den Bürgerkrieg beschränken müsste. Ich habe mich für einen Überblick über die Breite der bisherigen Forschung entschieden, nehme daher den skizzierten Nachteil in Kauf und gehe auf besondere Stärken oder Schwächen einzelner Arbeiten nur punktuell, nicht aber systematisch ein. ${ }^{8}$

\section{Während des Bürgerkrieges zu beeinflussende Faktoren}

In diesem Kapitel geht es um die Effekte des Kriegsendes, der Zahl der Kriegsopfer und der Kriegsdauer.

\section{Art des Kriegsendes}

Laut sieben von 15 Studien besteht nach Siegen eine statistisch signifikant geringere Wahrscheinlichkeit eines weiteren Bürgerkrieges als nach Friedensabkommen. ${ }^{9}$

7 Darin folgt der Bericht ein Stück weit dem methodisch anspruchsvolleren, aber auch deutlich längeren Aufsatz von Jeffrey Dixon (2009) über Bürgerkriegsursachen.

$8 \mathrm{Zu}$ meiner weiteren Entlastung weise ich darauf hin, dass fast alle der vorzustellenden Studien ein Begutachtungsverfahren durchlaufen haben und deshalb Mindeststandards erfüllen sollten.

9 Fortna (2003: 110; 2004: 274; 2008: 116); Kreutz (2010: 248); Licklider (1995: 685); Toft (2010 b: 9, 54-57). Mason et al. (2011: 183, 185) sehen diesen Effekt nur in den ersten Nachkriegsjahren. 
Der gegenteilige Schluss, dass nach Friedensabkommen seltener Rückfälle in den Krieg geschehen als nach Siegen, ist in der Forschung schwach vertreten. Nur eine Studie mit geringer Fallzahl (Meernik et al. 2010: 324) und eine deskriptive Statistik (Doyle/Sambanis 2006: 87) lassen sich hier anführen. Damit weist die Forschung zwar deutlich in eine Richtung, doch sieht ein erheblicher Teil einschlägiger Arbeiten keinen statistisch signifikanten Effekt von Siegen oder keine statistisch signifikant größere Wirkung des einen Kriegsendes im Vergleich zum anderen. ${ }^{10}$

Tabelle 1 fasst die Angaben zusammen. Wie in den folgenden Tabellen gibt eine Zeile an, wie viele Studien nur mit kleinen Einschränkungen oder Abweichungen zum entsprechenden Ergebnis kommen, etwa bei bestimmten Modellen oder Zeiträumen. Auch tauchen hier Studien auf, welche die abhängige Variable etwas anders fassen als die Arbeiten in der davorstehenden Zeile.

Tabelle 1: Die These, dass nach einem Sieg der Frieden statistisch signifikant stabiler sei als nach einem Friedensabkommen, ...

\begin{tabular}{|l|c|c|c|}
\hline & trifft zu & trifft nicht zu & $\begin{array}{c}\text { erlaubt keine } \\
\text { Aussage }\end{array}$ \\
\hline Studien & 6 & 1 & 6 \\
\hline Studien mit eingeschränkter Geltung & 1 & 1 & 0 \\
\hline Gesamt & 7 & 2 & 6 \\
\hline
\end{tabular}

Dass jede zweite Arbeit Siege für friedensverträglicher hält als Friedensabkommen, dürfte manche EntscheidungsträgerInnen in der Ansicht bestärken, von außen nicht möglichst schnell auf ein Friedensabkommen zu drängen, sondern einen Bürgerkrieg bis zum Sieg einer Seite ausbrennen zu lassen (vgl. »Give War a Chance«; Luttwak 1999). Roy Licklider (1995: 686) warnt vor diesem Schluss, da nach Siegen eine erhöhte Wahrscheinlichkeit eines Genozids bestehe. Der Literaturbericht stellt noch eine Reihe von Befunden vor, die Luttwaks Empfehlung widersprechen. Wie es darüber hinaus festzuhalten gilt, machen die zitierten Studien keine Aussagen

10 Gurses et al. (2008: 147); Mukherjee (2006 a: 422-424; 2006 b: 498); Pearson et al. (2006: 124); Quinn et al. (2007: 183-184); Walter (2004: 379-381). Es gibt keinen klaren Zusammenhang zwischen Befund und Vorgehen, wie eine Qualitative Comparative Analysis (Ragin 1987; Cronqvist 2007) zeigte, bei der ich die verschiedenen Arbeiten als Fälle und die Teilaspekte des jeweiligen Vorgehens als erklärende Faktoren betrachtet habe. Studien, die von der gleichen Definition von Bürgerkrieg ausgehen, in etwa die gleiche Fallzahl zugrunde legen oder die gleiche Methode der Datenanalyse verwenden, kommen dennoch zu unterschiedlichen Ergebnissen. Zwar bescheinigt keine der Arbeiten, die maximal die ersten fünf Jahre nach Kriegsende einbeziehen, Siegen eine statistisch signifikant größere rückfallhemmende Wirkung als Friedensabkommen, doch widersprechen sich die Befunde mit einem längeren Untersuchungszeitraum. 
über deterministische, sondern über probabilistische Kausalität. Diese erlauben keine klare Folgerung oder Handlungsempfehlung für den einzelnen Fall. Aus den gerade präsentierten Zahlen lässt sich eben nicht eindeutig ableiten, dass man in Syrien auf den Sieg einer Seite hinarbeiten sollte, um einen stabilen Frieden herbeizuführen. Ein entsprechender Vorbehalt gilt für alle noch zu skizzierenden praxeologischen Schlüsse.

\section{Kriegsopfer}

Die meisten Studien rechnen mit der absoluten Opferzahl, andere setzen diese in Verhältnis zur Einwohnerzahl oder Dauer des Krieges. Ich betrachte all diese Varianten so, als ob es sich um einen Ansatz handeln würde. Die absolute Mehrheit der ausgewerteten Studien kommt zum Schluss, dass mit statistischer Signifikanz eine umso höhere Wahrscheinlichkeit eines neuen Bürgerkrieges besteht, je mehr Opfer der vorangegangene Bürgerkrieg gefordert hat. Mehr als ein Drittel der Studien findet allerdings keine statistisch signifikante Wirkung vor; nur etwa jede zehnte hält eine hohe Opferzahl für rückfallhemmend. ${ }^{11}$

Tabelle 2: Eine höhere Zahl von Kriegsopfern ist...

\begin{tabular}{|l|c|c|c|}
\hline & $\begin{array}{c}\text { stat. signifikant } \\
\text { rückfallfördernd }\end{array}$ & $\begin{array}{c}\text { stat. signifikant } \\
\text { rückfallhemmend }\end{array}$ & $\begin{array}{c}\text { ohne stat. signifi- } \\
\text { kante Wirkung }\end{array}$ \\
\hline Studien & 13 & 3 & 7 \\
\hline Studien mit eingeschränkter Geltung & 4 & 0 & 3 \\
\hline Gesamt & 17 & 3 & 10 \\
\hline
\end{tabular}

Insgesamt stützen die statistischen Befunde die These, dass steigende Opferzahlen die Gräben zwischen den Konfliktparteien vertiefen und dadurch einen stabilen

11 Von einer statistisch signifikanten rückfallfördernden Wirkung berichten Chapman/Roeder (2007: 685); Fortna (2003: 110; 2004: 285) nach 1945; Glassmyer/Sambanis (2008: 372-374); Gurses et al. 2008: 147); Hartzell (2009: 359); Hartzell/Hoddie (2003: 328); Hartzell et al. (2001: 198); Mason et al. (2011: 186); Mattes/Savun (2009: 754); Mukherjee (2006a: 422-424; 2006 b: 498); Quinn et al. (2007: 185). Jarstad/Nilsson (2008: 216) und Nilsson (2008: 488) beobachten, dass nach Kriegen eher ein Rückfall droht als nach bewaffneten Konflikten. Doyle/Sambanis (2000: 788; 2006: 98) sehen ebenfalls eine rückfallfördernde Wirkung, beschränken ihre abhängige Variable aber nicht auf die Abwesenheit von Bürgerkrieg. Eine statistisch signifikante rückfallhemmende Wirkungen verzeichnen hingegen Collier (2000: 8), Meernik et al. (2010: 321) und Toft (2010 b: 56). Keinen statistisch signifikanten Effekt ermitteln DeRouen et al. (2009: 382), Falch (2006: 70), Fortna (2008: 116), Hartzell/Hoddie (2007: 78-79), Pearson et al. (2006: 121), Sambanis/Schulhofer-Wohl (2009: 108) und Walter (2004: 379-381). Zum gleichen Ergebnis kommen Collier et al. (2008: 467) für die ersten Nachkriegsjahre, Fortna (2004: 285) nur für den Zeitraum nach 1989 und Walter (2002: 86) mit Blick auf die Implementierung von Friedensabkommen. 
Frieden erschweren. Des Weiteren dürfte ein opferreicher Krieg mit größeren materiellen Verheerungen einhergehen als ein nicht so opferreicher Waffengang. Wie dieser Bericht noch darlegt, besteht bei einer schwachen Wirtschaft eine größere Wahrscheinlichkeit eines neuen Bürgerkrieges als bei einer starken.

Um die Wahrscheinlichkeit weiterer Bürgerkriege gering zu halten, gilt es, die Zahl ihrer Opfer zu minimieren. So klar sich dieses Ziel umreißen lässt, so schwer fällt es, einen Weg dorthin zu finden. Bedrohten Zivilisten während des Bürgerkrieges die Grenzen zu öffnen, kann viele Menschen retten. Auch dank humanitärer Hilfe überleben viele Menschen einen Bürgerkrieg. Hilfslieferungen können aber den Kriegsparteien Ressourcen verschaffen, den Krieg fortzuführen, womöglich bis zu solchen Phasen, in denen noch mehr Menschen sterben als zum gegebenen Zeitpunkt (vgl. Luttwak 1999: 40-42). Ein konsequent durchgesetztes, für alle Seiten geltendes Waffenembargo verhindert, dass die Konfliktparteien weitere Mittel für ihren Krieg erhalten. Es kann allerdings die Asymmetrie in der Bewaffnung der Kontrahenten festschreiben, die extrem einseitige Gewalt begünstigt, der dann vor allem ZivilistInnen zum Opfer fallen. Man denke an die Belagerer Sarajevos, die einige Tausend Geschütze auf den Bergen rund um die Stadt postiert hatten, während deren Verteidiger lange Zeit nur wenige, fast ausschließlich leichte Waffen besaßen. Eine andere Option für externe Akteure sind militärische Interventionen. Doch wie ein Aufsatz die bisherige Forschung resümiert, ziehen militärische Interventionen der Tendenz nach Bürgerkriege in die Länge (Regan 2010: 461, 463; siehe auch Cunningham 2010: 122).

\section{Kriegsdauer}

Nimmt man an, dass in längeren Kriegen mehr Menschen sterben als in kürzeren, sollten die Befunde zur Kriegsdauer die Ergebnisse zur Opferzahl widerspiegeln. Doch es zeigt sich ein anderes Bild.

Tabelle 3: Dauerte der letzte Bürgerkrieg lange, so ist dies...

\begin{tabular}{|l|c|c|c|}
\hline & $\begin{array}{c}\text { stat. signifikant } \\
\text { rückfallfördernd }\end{array}$ & $\begin{array}{c}\text { stat. signifikant } \\
\text { rückfallhemmend }\end{array}$ & $\begin{array}{c}\text { ohne stat. signifi- } \\
\text { kante Wirkung }\end{array}$ \\
\hline Studien & 3 & 6 & 13 \\
\hline Studien mit eingeschränkter Geltung & 0 & 1 & 3 \\
\hline Gesamt & 3 & 7 & 16 \\
\hline
\end{tabular}


Die wenigsten Studien schreiben einer längeren Kriegsdauer eine statistisch signifikante rückfallfördernde Wirkung zu. ${ }^{12}$ Schon mehr Arbeiten sehen im Gegenteil einen statistisch signifikanten rückfallhemmenden Effekt, ${ }^{13}$ die absolute Mehrheit jedoch findet keinerlei statistisch signifikante Wirkung vor. ${ }^{14}$ Dieser Befund steht dann im Einklang mit dem Ergebnis zur Opferzahl, wenn lange Kriege überproportional häufig mit geringer Intensität ausgetragen werden.

Vermutlich stellen die meisten Studien keine statistisch signifikante Wirkung fest, weil lange Kriege widersprüchliche Folgen haben. Einerseits zementieren sie die Feindschaft zwischen den Konfliktparteien und stärken die Position von Gewaltakteuren. Andererseits verursachen lange Kriege eine größere Kriegsmüdigkeit und beseitigen eher als kurze Kriege Illusionen über die eigenen militärischen Fähigkeiten.

Da die Befunde zu den Wirkungen der Kriegsdauer nicht klar in eine Richtung zeigen, untermauern sie nicht die Forderung, Bürgerkriege um eines stabileren Friedens willen ausbluten zu lassen.

\section{Kaum oder nicht zu beeinflussende Merkmale des Bürgerkrieges}

Im Folgenden präsentiere ich die Befunde zu den Wirkungen des Konflikttyps und der Zahl der Konfliktparteien.

\section{Konflikttyp}

Viele Arbeiten stellen Konflikte zwischen ethnisch definierten Parteien anderen Konstellationen gegenüber. Manche unterscheiden Identitätskonflikte von anderen Auseinandersetzungen und meinen mit Identität vor allem askriptive Identität, die sich, wenn überhaupt, viel schwieriger wechseln lässt als eine Identität, die sich über Ideologie oder materielle Interessen definiert. Da solche Konfliktformationen sta-

12 Hartzell (2009: 359); Meernik et al. (2010: 321); Nilsson (2008: 488).

13 Chapman/Roeder (2007: 685); Fortna (2008: 116); Hartzell et al. (2001: 198); Mason et al. (2011: 185); Quinn et al. (2007: 185); Walter (2004: 379-381). Fortna (2004: 285) sieht eine statistisch signifikante rückfallhemmende Wirkung nur bei Fällen nach 1989.

14 Falch (2006: 70); Fortna (2003: 110); Gurses et al. (2008: 147); Hartzell/Hoddie (2003: 328; 2007 : 78-79); Jarstad/Nilsson (2008: 216); Mattes/Savun (2009: 754); Mukherjee (2006 a: 422-424; 2006 b: 498); Pearson et al. (2006: 121, 124); Toft (2010b: 56); Sambanis (2000: 466-467, 470); Walter (2002: 86). Ebenfalls keinen statistisch signifikanten Befund sieht Nilsson (2008: 489) mit Blick auf neue Gewalt innerhalb einer Konfliktdyade. Ebenso wenig findet Fortna (2004: 285) für den gesamten Zeitraum nach 1945 einen statistisch signifikanten Effekt vor. Gleiches gilt für Doyle/ Sambanis (2000: 788), welche die abhängige Variable nicht auf die Abwesenheit eines Bürgerkrieges beschränken. 
biler sind als andere, könnte man annehmen, dass sie eher oder häufiger erneut zu einem Bürgerkrieg eskalieren.

Fasst man Konflikte entlang askriptiver Identität im Allgemeinen und ethnische Konflikte im Besonderen zu einem Konflikttyp zusammen, zeigt sich, dass dessen Vorliegen einen dauerhaften Frieden nicht wahrscheinlicher macht. Tabelle 4 zeigt dies. Keine der ausgewerteten Studien schreibt diesem Konflikttyp eine statistisch signifikante rückfallhemmende Wirkung zu. Allerdings gibt es mehr Arbeiten, die keinen statistisch signifikanten Effekt feststellen, als solche, welche von einer statistisch signifikanten rückfallfördernden Wirkung ausgehen. ${ }^{15}$

Tabelle 4: Fand der letzte Bürgerkrieg zwischen Ethnien statt, so ist dies... ${ }^{16}$

\begin{tabular}{|l|c|c|c|}
\hline & $\begin{array}{c}\text { stat. signifikant } \\
\text { rückfallfördernd }\end{array}$ & $\begin{array}{c}\text { stat. signifikant } \\
\text { rückfallhemmend }\end{array}$ & $\begin{array}{c}\text { ohne stat. signifi- } \\
\text { kante Wirkung }\end{array}$ \\
\hline Studien & 6 & 0 & 12 \\
\hline Studien mit eingeschränkter Geltung & 4 & 0 & 2 \\
\hline Gesamt & 10 & 0 & 14 \\
\hline
\end{tabular}

Die Zahlen lesen sich durchaus als Plädoyer für Versuche, etwaige askriptive Identitäten der Konfliktparteien zu relativieren. Jedoch ist Skepsis darüber geboten,

15 Die Frage, ob nach ethnischen Kriegen eher ein Rückfall droht als nach anderen, ist nicht die gleiche wie die, ob bei hoher ethnischer Fragmentierung oder Polarisierung ein Bürgerkrieg wahrscheinlicher ist als bei einer geringen. Von den Arbeiten zum Rückfall in den Bürgerkrieg sehen Glassmyer/Sambanis (2008: 372-374), Sambanis/Schulhofer-Wohl (2009: 108) und Mason et al. (2011: 184-185) eine statistisch signifikante rückfallfördernde Wirkung; die Letztgenannten gehen allerdings von einem nicht-linearen Zusammenhang aus. Sambanis (2000: 466-467) findet eine statistisch signifikante rückfallhemmende Wirkung hoher ethnischer Fragmentierung vor. Keinen statistisch signifikanten Effekt stellen fest: Collier et al. (2008: 471-472); Falch (2006: 70); Mukherjee (2006a: 422-424; 2006 b: 498). Doyle/Sambanis (2006: 96) sehen ebenso keinen solchen Effekt, beschränken die abhängige Variable aber nicht auf die Abwesenheit eines neuen Bürgerkrieges.

16 Auf eine statistisch signifikante rückfallfördernde Wirkung stoßen Falch (2006: 70, 73), Gurses et al. (2008: 147), Kreutz (2010: 248), Mason et al. (2011: 184, 186), Mattes/Savun (2009: 754) und Sambanis/Schulhofer-Wohl (2009: 108). Doyle/Sambanis (2008: 788; 2006: 96) stellen ebenfalls einen solchen Effekt fest, doch umfasst bei ihnen die abhängige Variable mehr als nur das Ausbleiben eines weiteren Krieges. Bei Hartzell/Hoddie (2003: 328) zeigt sich nur eine statistisch signifikante rückfallfördernde Wirkung, wenn sie das Weibull-Modell zugrunde legen. Bei Licklider (1995: 686-687) sind Friedensabkommen nach solchen Konflikten instabiler. Keine statistisch signifikante Wirkung finden: Fortna (2003: 110; 2004: 285; 2008: 116); Glassmyer/Sambanis (2008: 372-374); Hartzell (1999: 16-17); Hartzell et al. (2001: 189-190); Hartzell/Hoddie (2007: 78-79); Mukherjee (2006a: 422-424; 2006 b: 498); Pearson et al. (2006: 121-124); Sambanis (2000: 466-467, 471); Toft (2010b: 56). Bei Hartzell/Hoddie (2007: 328) zeigt sich mit dem Cox-Modell keine statistisch signifikante Wirkung. Gleiches gilt für »ethnische Revolutionen« und »ethnische Sezessionen« bei Quinn et al. (2007: 184). 
ob sich solche Identitäten in oder nach einem Bürgerkrieg ändern lassen. Externe Akteure können zwar die Identitäten der Konfliktparteien bestärken, wenn sie deren Selbstbeschreibungen aufgreifen. Bei aller Wandelbarkeit der Inhalte und Bedeutungen von Identitäten bedeutet dies aber nicht, dass Außenstehende den Konflikttyp zu wandeln vermögen, indem sie die Selbstdefinitionen der Kontrahenten zurückweisen. Während eines Bürgerkrieges spielen die Selbstzuschreibung und die Fremdzuschreibung durch den Feind eine weit wichtigere Rolle als die Kategorisierungen externer Mächte (vgl. Kaufmann 1996: 139-143).

\section{Zahl der Konfliktparteien}

Mit einer größeren Zahl der Konfliktparteien sind mehr Anliegen gegeneinander auszubalancieren. Die Nachkriegsordnung ist dann komplexer und vermutlich auch instabiler als in Konstellationen mit wenigen Kontrahenten. Von einer größeren Zahl an Konfliktparteien geht aber, so der Befund von zwei Dritteln der Arbeiten, keine statistisch signifikante rückfallhemmende Wirkung aus. ${ }^{17}$ Drei Studien attestieren einer größeren Zahl von Konfliktparteien zumindest eingeschränkt eine statistisch signifikante rückfallfördernde Wirkung. ${ }^{18}$

Tabelle 5: Eine größere Zahl von Konfliktparteien ist...

\begin{tabular}{|l|c|c|c|}
\hline & $\begin{array}{c}\text { stat. signifikant } \\
\text { rückfallfördernd }\end{array}$ & $\begin{array}{c}\text { stat. signifikant } \\
\text { rückfallhemmend }\end{array}$ & $\begin{array}{c}\text { ohne stat. signifi- } \\
\text { kante Wirkung }\end{array}$ \\
\hline Studien & 0 & 0 & 4 \\
\hline Studien mit eingeschränkter Geltung & 3 & 0 & 3 \\
\hline Gesamt & 3 & 0 & 7 \\
\hline
\end{tabular}

Die Protagonisten selbst bestimmen die Zahl der Konfliktparteien, indem sie sich zum Beispiel aus dem Konflikt zurückziehen. Externe Akteure können zwar durch massives Eingreifen eine Kriegspartei aus dem Konflikt nehmen oder eine neue Kriegspartei ins Feld führen. All dies betrifft aber in erster Linie den kämpfenden

17 Fortna (2003: 110; 2004: 285; 2008: 106); Sambanis/Schulhofer-Wohl (2009: 108). Bei Glassmyer/ Sambanis (2008: 369-371) verliert der Faktor nach zwei Jahren seine statistische Signifikanz. In Doyle/Sambanis (2000: 788) ist die Zahl der Konfliktparteien in zwei von drei Modellen ohne statistisch signifikante Wirkung, in Doyle/Sambanis (2006: 191) in einem von zehn Modellen.

18 Bei Glassmyer/Sambanis (2008: 369-371) geht von einer größeren Zahl an Konfliktparteien nur in den ersten beiden Nachkriegsjahren eine statistisch signifikante rückfallfördernde Wirkung aus, danach nicht mehr. Doyle/Sambanis (2000: 78) stoßen in einem von drei Modellen auf einen statistisch signifikanten destruktiven Effekt, bei Doyle/Sambanis (2006: 101) trifft dies auf neun von zehn Modellen zu. Allerdings beschränken sie die abhängige Variable nicht auf die Abwesenheit eines neuen Bürgerkrieges. 
Teil einer Konfliktpartei, weit weniger aber die Gruppen, die hinter ihren KämpferInnen stehen.

\section{Nach dem Krieg beeinflussbare Aspekte der Nachkriegsordnung}

Das folgende Kapitel stellt dar, wie sich eine Machtteilung der Konfliktparteien, eine Teilung des umstrittenen Staates, die Regimeform, die Präsenz von Friedenstruppen und die Größe staatlicher Streitkräfte auf die Wahrscheinlichkeit eines weiteren Bürgerkrieges auswirken.

\section{Machtteilung}

Bei einer Machtteilung regieren die Konfliktparteien gemeinsam; jede von ihnen kontrolliert einen Teil der staatlichen Institutionen oder des Territoriums. Die Machtteilung soll den Konfliktparteien garantieren, dass die andere Seite die staatlichen Zwangsmittel nicht dazu nutzen kann, den Bürgerkrieg nachträglich zu gewinnen (Hartzell/Hoddie 2007: 12). Die Machtteilung schützt vor Exklusion, die in der allgemeinen Bürgerkriegsursachenforschung als kriegstreibend gilt. ${ }^{19}$

Etwas mehr als die Hälfte der ausgewerteten Studien bescheinigt der Machtteilung, die Kriegswahrscheinlichkeit statistisch signifikant zu verringern. ${ }^{20}$ Eine statistisch signifikante rückfallfördernde Wirkung sieht nur die bereits erwähnte Arbeit, die einen zu weiten Begriff von Machtteilung zugrunde legt (Mukherjee 2006 b: 498). Fast jede zweite Studie ermittelt keinen statistisch signifikanten Effekt. ${ }^{21}$ Die Befunde hängen stark davon ab, welche Arten des Kriegsendes die Fallauswahl der jeweiligen Studien berücksichtigt. Einen statistisch signifikanten rückfallhemmenden Effekt der Machtteilung konstatieren vor allem jene Arbeiten, die ausschließlich Friedensabkommen miteinander vergleichen. ${ }^{22}$ Keine statistisch signifikante Wirkung der Machtteilung sehen demgegenüber gerade solche Studien, die neben Friedensabkommen auch andere Arten des Kriegsendes einbeziehen. ${ }^{23}$

19 Cederman et al. (2011); Gottwald (2009); Regan/Norton (2005); Wimmer et al. (2009).

20 DeRouen et al. (2009: 382); Glassmyer/Sambanis (2008: 372-374); Hartzell (1999: 16-17); Hartzell/Hoddie (2003: 328; 2007: 78-79); Hartzell et al. (2001: 199); Mattes/Savun (2009: 754); Mukherjee (2006 b: 498); Jarstad/Nilsson (2008: 216); Nilsson (2008: 488); Walter (2002: 83-84).

21 Chapman/Roeder (2007: 685); Collier et al. (2008: 471); DeRouen et al. (2009: 382); Falch (2006: 70); Jarstad/Nilsson (2008: 21); Mattes/Savun (2009: 754); Mukherjee (2006b: 498); Pearson et al. (2006: 121).

22 DeRouen et al. (2009); Hartzell (1999); Hartzell/Hoddie (2003; 2007); Jarstad/Nilsson (2008); Mattes/Savun (2009); Nilsson (2008); Walter (2002).

23 Chapman/Roeder (2007); Collier et al. (2008); Falch (2006); Glassmyer/Sambanis (2008); Mukherjee (2006 b); Pearson et al. (2006). 
Tabelle 6: Eine Machtteilung zwischen den früheren Kriegsparteien ist... ${ }^{24}$

\begin{tabular}{|l|c|c|c|}
\hline & $\begin{array}{c}\text { stat. signifikant } \\
\text { rückfallfördernd }\end{array}$ & $\begin{array}{c}\text { stat. signifikant } \\
\text { rückfallhemmend }\end{array}$ & $\begin{array}{c}\text { ohne stat. signifi- } \\
\text { kante Wirkung }\end{array}$ \\
\hline Studien & 0 & 6 & 1 \\
\hline Studien mit eingeschränkter Geltung & 1 & 5 & 8 \\
\hline Gesamt & 1 & 11 & 9 \\
\hline
\end{tabular}

Da Machtteilung vor allem nach Friedensabkommen zum Einsatz kommt, stehen die Befunde nicht im Einklang mit der Folgerung, nach Siegen sei ein neuer Bürgerkrieg unwahrscheinlicher als nach Abkommen. Auch das spricht gegen den Rat »Give War a Chance«.

In Tabelle 6 stechen die großen Anteile der Studien mit eingeschränkter Geltung ins Auge. Daher lohnt es sich, die Befunde nach Varianten der Machtteilung zu differenzieren. Eine politische Machtteilung liegt vor, wenn alle früheren Kriegsparteien an der Regierung beteiligt sind. Der politischen Machtteilung schreibt lediglich die problematische Arbeit von Mukherjee (2006 b: 498) eine statistisch signifikante Wirkung zu, aber auch nur nach Kriegen, die in einem militärischen Patt endeten. Vier Studien werten die politische Machtteilung als statistisch signifikant rückfallhemmend ${ }^{25}$ fünf messen ihr keinen statistisch signifikanten Effekt bei. ${ }^{26}$

Tabelle 7: Eine politische Machtteilung zwischen den früheren Kriegsparteien ist...

\begin{tabular}{|l|c|c|c|}
\hline & $\begin{array}{c}\text { stat. signifikant } \\
\text { rückfallfördernd }\end{array}$ & $\begin{array}{c}\text { stat. signifikant } \\
\text { rückfallhemmend }\end{array}$ & $\begin{array}{c}\text { ohne stat. signifi- } \\
\text { kante Wirkung }\end{array}$ \\
\hline Studien & 0 & 2 & 4 \\
\hline Studien mit eingeschränkter Geltung & 1 & 2 & 1 \\
\hline Gesamt & 1 & 4 & 5 \\
\hline
\end{tabular}

In die Kategorie der territorialen Machtteilung fallen Arrangements von regionaler Autonomie bis zu föderalistischen Systemen. Eine knappe Mehrheit der Stu-

24 Da der Bericht noch näher auf einzelne Befunde zur Machtteilung eingeht, erläutere ich an dieser Stelle nicht, was hinter den Studien mit eingeschränkter Geltung steht.

25 Glassmyer/Sambanis (2008: 372-374) und Mattes/Savun (2009: 754) attestieren der politischen Machtteilung einen statistisch signifikanten rückfallhemmenden Effekt. Das Gleiche gilt für Mukherjee (2006b: 498), sofern diese Machtteilung auf den militärischen Sieg einer Seite folgt. Walter (2002: 84-88) stellt ebenso einen positiven Effekt der politischen Machtteilung auf die Implementierung eines Friedensabkommens fest.

26 DeRouen et al. (2009: 382); Falch (2006: 70); Hartzell/Hoddie (2007: 170); Jarstad/Nilsson (2008: 216). Für sich allein genommen und in Kombination mit UN-Friedenstruppen geht von der politischen Machtteilung bei Mukherjee (2006 b: 498) keine statistisch signifikante Wirkung aus. 
dien attestiert der territorialen Machtteilung eine statistisch signifikante rückfallhemmende Wirkung, alle anderen sehen keinen statistisch signifikanten Effekt.

Tabelle 8: Eine territoriale Machtteilung zwischen den früheren Kriegsparteien ist ... ${ }^{27}$

\begin{tabular}{|l|c|c|c|}
\hline & $\begin{array}{c}\text { stat. signifikant } \\
\text { rückfallfördernd }\end{array}$ & $\begin{array}{c}\text { stat. signifikant } \\
\text { rückfallhemmend }\end{array}$ & $\begin{array}{c}\text { ohne stat. signifi- } \\
\text { kante Wirkung }\end{array}$ \\
\hline Studien & 0 & 5 & 5 \\
\hline Studien mit eingeschränkter Geltung & 0 & 1 & 0 \\
\hline Gesamt & 0 & 6 & 5 \\
\hline
\end{tabular}

Bei der militärischen Machtteilung nehmen die staatlichen Sicherheitskräfte Truppen der Rebellen in ihre Reihen auf; in Ausnahmefällen behalten alle Kriegsparteien separate Streitkräfte (Hartzell/Hoddie 2007: 32). Die Mehrzahl der Studien stellt keine statistisch signifikante Wirkung fest, die restlichen erachten die militärische Machtteilung für statistisch signifikant rückfallhemmend. ${ }^{28}$

Tabelle 9: Eine militärische Machtteilung zwischen den früheren Kriegsparteien ist...

\begin{tabular}{|l|c|c|c|}
\hline & $\begin{array}{c}\text { stat. signifikant } \\
\text { rückfallfördernd }\end{array}$ & $\begin{array}{c}\text { stat. signifikant } \\
\text { rückfallhemmend }\end{array}$ & $\begin{array}{c}\text { ohne stat. signifi- } \\
\text { kante Wirkung }\end{array}$ \\
\hline Studien & 0 & 1 & 2 \\
\hline Studien mit eingeschränkter Geltung & 0 & 2 & 3 \\
\hline Gesamt & 0 & 3 & 5 \\
\hline
\end{tabular}

Wie auffällt, kommt nur bei der territorialen Machtteilung eine absolute Mehrheit der Studien zum Schluss, dass diese einen weiteren Bürgerkrieg in statistisch signifikanter Weise unwahrscheinlicher macht. Rechnet man bei den drei Varianten

27 Statistisch signifikant rückfallhemmend wirkt sie laut DeRouen et al. (2009: 382), Glassmyer/ Sambanis (2008: 372-374), Hartzell/Hoddie (2007: 170), Hartzell et al. (2001: 199) und Jarstad/ Nilsson (2008: 216). Bei Walter (2002: 84-88) fördert sie die Umsetzung von Friedensabkommen. Keinen statistisch signifikanten Effekt gibt es laut Chapman/Roeder (2007: 685), Collier et al. (2008: 471), Falch (2006: 70), Mattes/Savun (2009: 754) sowie Pearson et al. (2006: 121).

28 DeRouen et al. (2009: 382) sehen eine statistisch signifikante rückfallhemmende Wirkung, das Gleiche gilt für Glassmyer/Sambanis (2008: 372-374), die ihren Befund aber auf die ersten beiden Nachkriegsjahre beschränken. Jarstad/Nilsson (2008: 216) bescheinigen der implementierten militärischen Machtteilung eine statistisch signifikante rückfallhemmende Wirkung, die bloße Vorkehrung einer solchen habe aber keinen statistisch signifikanten Effekt. Keine statistische Signifikanz erreicht dieser Faktor auch bei Hartzell/Hoddie (2007: 170-171), Mattes/Savun (2009: 754) und Glassmyer/Sambanis (2008: 372-374), wenn diese nicht nur die ersten beiden Nachkriegsjahre betrachten. Walter (2002: 88) attestiert der militärischen Machtteilung keine statistisch signifikante Wirkung auf die Umsetzung von Friedensabkommen. 
der Machtteilung die Zahl der Studien je Wirkungsrichtung zusammen, weicht deren jeweilige Summe von den Zahlen in Tabelle 6 zur Machtteilung allgemein ab. Dies liegt daran, dass nur ein Teil der Arbeiten die Formen der Machtteilung getrennt untersucht (z.B. Jarstad/Nilsson 2008).

\section{Teilung des Staates}

Die Mühen der Machtteilung lassen sich umgehen, wenn man die Konfliktparteien voneinander separiert und ihnen jeweils einen eigenen Staat gibt. Chaim Kaufmann (1996: 170) sieht nach Kriegen zwischen ethnisch definierten Gruppen die Teilung als schlechteste Lösung, abgesehen von allen anderen Optionen. Die konsequente Separation der verfeindeten Ethnien und die Teilung des Staates eröffneten oftmals die einzige Möglichkeit, das Sicherheitsdilemma zwischen den Konfliktparteien zu beseitigen und den Frieden zu sichern (Kaufmann 1996; 1998). Gegner dieser Option halten weitgehende Separationen ohne Verbrechen für nicht durchführbar, sehen durch eine Teilung den interethnischen Konflikt nur auf die internationale Ebene verschoben und fürchten, Teilungen könnten dritte Staaten destabilisieren (Fearon 2004; Horowitz et al. 2009; Kumar 1997).

Tabelle 10: Eine Separation der Konfliktparteien und Teilung umstrittener Staaten sind...

\begin{tabular}{|l|c|c|c|}
\hline & $\begin{array}{c}\text { stat. signifikant } \\
\text { rückfallfördernd }\end{array}$ & $\begin{array}{c}\text { stat. signifikant } \\
\text { rückfallhemmend }\end{array}$ & $\begin{array}{c}\text { ohne stat. signifi- } \\
\text { kante Wirkung }\end{array}$ \\
\hline Studien & 1 & 1 & 3 \\
\hline Studien mit eingeschränkter Geltung & 0 & 1 & 1 \\
\hline Gesamt & 1 & 2 & 4 \\
\hline
\end{tabular}

Barbara Walter (2004: 379, 391) konstatiert nach Teilungen eine statistisch signifikant erhöhte Wahrscheinlichkeit eines weiteren Bürgerkrieges. Dagegen halten Thomas Chapman und Philip Roeder (2007: 685) eine Teilung für statistisch signifikant rückfallhemmend, untersuchen allerdings nur die ersten beiden Nachkriegsjahre. Carter Johnson (2008: 160-163) sieht eine statistisch signifikant verringerte Kriegswahrscheinlichkeit, sobald die verfeindeten Ethnien konsequent voneinander getrennt sind. Drei Studien kommen zu keinem statistisch signifikanten Befund. ${ }^{29}$ Chapman und Roeder (2007: 685) stellen ebenfalls keinen solchen Effekt fest, wenn zwar die verfeindeten Gruppen voneinander getrennt werden, der umstrittene Staat aber ungeteilt bleibt.

29 Mukherjee (2006 a: 422-424); Sambanis (2000: 466-467, 469); Sambanis/Schulhofer-Wohl (2009: 108). 
Die im doppelten Sinn geringe Fallzahl warnt vor voreiligen Schlüssen. Zum einen liegen nur wenige quantitative Studien zu diesem Faktor vor, zum anderen liefen in den letzten Jahrzehnten nur wenige Bürgerkriege auf eine Teilung hinaus. Schon ein einziger falsch zugeordneter Fall wirkt sich stark auf die Ergebnisse aus (Sambanis/Schulhofer-Wohl 2009: 88-90). Zudem gilt es zu beachten, dass Tabelle 10 nur die Wahrscheinlichkeit eines weiteren Bürgerkrieges berücksichtigt, nicht aber die eines internationalen Krieges zwischen den Staaten, die durch die Teilung entstehen. ${ }^{30}$

\section{Regimeform}

Der Theorie nach reduziert Demokratie Konfliktursachen (Wolff: 2008: 45-47), insofern sie die Regierung dazu bringt, die Probleme der BürgerInnen nicht zu ignorieren (Huntington 1996: 12-13; Sen 1999: 7-8). Zudem entschärfen demokratische Verfahren Konflikte, weil sie Bahnen für die gewaltlose Austragung bereitstellen (Gurr 2000: 85-88; Przeworski 1999). Schaut man auf den Effekt der Demokratie in Nachbürgerkriegsgesellschaften, fällt das Urteil nicht allzu sehr zugunsten dieser Herrschaftsform aus. Drei Arbeiten, von denen sich zwei weitgehend decken, sehen bei einer Demokratie eine verringerte Wahrscheinlichkeit eines neuen Bürgerkrieges (Gurses et al. 2008: 147; Mukherjee 2006 a: 422-424; 2006 b: 498). Eine weitere Arbeit ermittelt in Demokratien eine geringere Rückfallwahrscheinlichkeit als in Semi-Demokratien (Walter 2004: 384). Fünf Studien sehen bei einer Demokratie oder größeren Nähe zur Demokratie keinen statistisch signifikanten Effekt auf die Wahrscheinlichkeit eines neuen Krieges. ${ }^{31}$ Paul Collier und seine Ko-AutorInnen (2008: 470) kommen zum Schluss, dass in Autokratien die Wahrscheinlichkeit eines weiteren Bürgerkrieges statistisch signifikant geringer ist als in der Restkategorie mit Demokratien und Anokratien, also Regimen im Graubereich zwischen Autokratie und Demokratie. ${ }^{32}$

30 Chapman/Roeder (2007: 688-689) stellen fest, dass Staaten, die aus Teilungen hervorgegangen sind, sich nicht wahrscheinlicher an einem internationalen Krieg beteiligen als andere. Dieser Befund widerlegt aber nicht die Befürchtung, dass eine Teilung zwar einen neuen Krieg zwischen den Kontrahenten innerhalb eines Staates ausschließt, doch einen internationalen Krieg zwischen den Konfliktparteien wahrscheinlicher macht.

31 DeRouen et al. (2009: 382); Fortna (2008: 116); Meernik et al. (2010: 323); Quinn et al. (2007: 185); Walter (2004: 384). Hinzu kommt der Befund von Walter (2002: 88), dass Einschränkungen der Exekutive, wie üblich in der Demokratie, die Umsetzung eines Friedensabkommens nicht statistisch signifikant wahrscheinlicher machen.

32 Siehe Polity IV: http://www.systemicpeace.org/polity/polity4.htm; und http://www.systemicpe ace.org/inscr/p4manualv 2009.pdf; 8.8.2012. 
Tabelle 11: Setzt eine Nachkriegsgesellschaft möglichst viele Prinzipien der Demokratie um, so ist dies...

\begin{tabular}{|l|c|c|c|}
\hline & $\begin{array}{c}\text { stat. signifikant } \\
\text { rückfallfördernd }\end{array}$ & $\begin{array}{c}\text { stat. signifikant } \\
\text { rückfallhemmend }\end{array}$ & $\begin{array}{c}\text { ohne stat. signifi- } \\
\text { kante Wirkung }\end{array}$ \\
\hline Studien & 0 & 3 & 5 \\
\hline Studien mit eingeschränkter Geltung & 1 & 1 & 1 \\
\hline Gesamt & 1 & 4 & 6 \\
\hline
\end{tabular}

Aus diesen Zahlen leitet sich kein Auftrag ab, mittels Demokratisierung den Frieden zu festigen. Der Zweifel an der Demokratisierung als Friedensstrategie verstärkt sich sogar noch angesichts dessen, dass eine solche Politik Nachbürgerkriegsgesellschaften in die turbulente Zone der Anokratien zu führen droht. Die allgemeine Forschung zu Bürgerkriegsursachen hat Anokratien als besonders bürgerkriegsgefährdet identifiziert. ${ }^{33}$ Anokratien, so die Erklärung, fehle die Fähigkeit der Repression, wie sie in Autokratien den innerstaatlichen Frieden erzwinge. Zugleich mangle es Anokratien am Potenzial institutioneller Schlichtung, das Demokratien auszeichne (Gates et al. 2001: 185-187). ${ }^{34}$ Allerdings können die Studien zum Rückfall in den Bürgerkrieg den klaren Trend der Arbeiten zu Bürgerkriegsursachen nicht bestätigen. Es überwiegt der Befund, dass von der Anokratie kein statistisch signifikanter Effekt ausgeht. ${ }^{35}$

Die Befunde zur Wirkung der Demokratie sprechen nicht für die Demokratisierung, doch sprechen die festgestellten Effekte der Anokratie auch nicht klar gegen diese Friedensstrategie. Wer auf den Aufbau einer Demokratie als Mittel der Friedenskonsolidierung verzichten möchte, sollte zudem folgenden Punkt zur Kenntnis nehmen: Arbeiten, die intensiv die Gefahren der Demokratisierung nach Bürgerkriegen untersuchen, verwerfen diese Friedensstrategie keineswegs (Gromes 2007:

33 Siehe z.B. Bleaney/Dimico (2011: 149); Hegre et al. (2001); zahlreiche Literaturverweise bei Spanger/Schesterinina (2012).

34 Ein Teil der Arbeiten, die Anokratien eine höhere Bürgerkriegswahrscheinlichkeit als Autokratien und Demokratien bescheinigen, nutzt Daten, die Anokratie auch über das Ausmaß innerstaatlicher Gewalt operationalisieren (Vreeland 2008). Inwiefern das die Ergebnisse verzerrt, ist strittig: siehe Spanger/Schesterinina (2012: 14-15).

35 Falch (2006: 70); Meernik et al. (2010: 323). Jarstad/Nilsson (2008: 216) sehen in zwei von drei Modellen keinen statistisch signifikanten Effekt. Gleiches gilt für Mason et al. (2011: 184, 186), wenn sie mit den einfachen Polity-Werten rechnen. Walter (2004: 384) ermittelt indirekt den Effekt von Anokratien, indem sie bei »klaren Autokratien« und »klaren Demokratien« keine statistisch signifikant geringere Kriegswahrscheinlichkeit als in der Restkategorie feststellt. Einen statistisch signifikant rückfallfördernden Effekt der Anokratie gibt es in einem Modell bei Mason et al. (2011: 184, 186), eine statistisch signifikant rückfallhemmende Wirkung bei einem Modell von Jarstad/ Nilsson (2008: 216). 
70-94; Paris 2004: 159-175). Zum einen, so legen sie nahe, ließen sich die Gefahren der Demokratisierung im Zaum halten, zum anderen gebe es nur schlechtere Alternativen zum Aufbau einer Demokratie.

Tabelle 12: Eine Anokratie ist...

\begin{tabular}{|l|c|c|c|}
\hline & $\begin{array}{c}\text { stat. signifikant } \\
\text { rückfallfördernd }\end{array}$ & $\begin{array}{c}\text { stat. signifikant } \\
\text { rückfallhemmend }\end{array}$ & $\begin{array}{c}\text { ohne stat. signifi- } \\
\text { kante Wirkung }\end{array}$ \\
\hline Studien & 0 & 0 & 2 \\
\hline Studien mit eingeschränkter Geltung & 1 & 1 & 3 \\
\hline Gesamt & 1 & 1 & 5 \\
\hline
\end{tabular}

\section{Friedenstruppen}

Das Ende des Ost-West-Konflikts eröffnete gerade den Vereinten Nationen neue Möglichkeiten, mit Friedenstruppen weitere Bürgerkriege zu verhindern. Friedenstruppen sollen die Demobilisierung der rivalisierenden Streitkräfte gewährleisten und Angriffe einer Seite auf die andere verhindern (Walter 2002: 20-40).

Laut 16 von 30 Studien geht von Friedenstruppen in statistisch signifikanter Weise eine rückfallhemmende Wirkung aus. ${ }^{36}$ Nur eine Arbeit schreibt Friedenstruppen einen statistisch signifikanten rückfallfördernden Effekt zu und beschränkt diesen Befund auf Friedenstruppen, die nicht von den Vereinten Nationen entsandt werden (Nilsson 2008: 489). 13 Studien stellen keinen statistisch signifikanten Effekt fest. ${ }^{37}$

Verglichen mit anderen nach dem Krieg gestaltbaren Faktoren, zeigen die Befunde zu den Friedenstruppen in eine klare Richtung. Angesichts dieser Zahlen fällt die allgemeine Empfehlung leicht, in Nachbürgerkriegsgesellschaften Friedenstruppen zu stationieren.

36 Dobbins et al. (2005: 247-248); Fortna (2004: 285; 2008: 116); Hartzell et al. (2001: 199); Hartzell/ Hoddie (2003: 328; 2007: 78-79); Hofmann/Schaffer (2009: 321); Kreutz (2010: 248); Mason et al. (2011: 185-187); Mattes/Savun (2009: 754); Pearson et al. (2006: 120); Walter (2002: 83-84). Collier et al. (2008: 473) halten höhere Ausgaben für Friedenstruppen für friedensfördernd. Fortna (2003: 110) nimmt Friedenstruppen mit einem Mandat nach Kapitel VII der UN-Charta aus diesem Befund aus. Bei Glassmyer/Sambanis (2008: 372-374) zeigt sich nur in fünf von sieben Modellen eine statistisch signifikante rückfallhemmende Wirkung; Quinn et al. (2007: 183-184) stellen eine solche nach Friedensabkommen fest.

37 Chapman/Roeder (2007: 685); Falch (2006: 70); Hartzell (1999: 16-17); Jarstad/Nilsson (2008: 216); Mukherjee (2006 a: 422-424; 2006 b: 498); Nilsson (2008: 488-489); Sambanis/SchulhoferWohl (2009: 108); Toft (2010b: 59-60). Bei Gurses et al. (2008: 147) und Meernik et al. (2010: 321) gibt es keinen statistisch signifikanten Effekt bei Friedenstruppen der Vereinten Nationen. Fortna (2003: 110) sieht keine statistisch signifikante Wirkung von Missionen nach Kapitel VII der UN-Charta, Fortna (2008: 116) hingegen bei Beobachter- und Interpositionsmissionen. 
Tabelle 13: Friedenstruppen sind...

\begin{tabular}{|l|c|c|c|}
\hline & $\begin{array}{c}\text { stat. signifikant } \\
\text { rückfallfördernd }\end{array}$ & $\begin{array}{c}\text { stat. signifikant } \\
\text { rückfallhemmend }\end{array}$ & $\begin{array}{c}\text { ohne stat. signifi- } \\
\text { kante Wirkung }\end{array}$ \\
\hline Studien & 0 & 12 & 9 \\
\hline Studien mit eingeschränkter Geltung & 1 & 4 & 4 \\
\hline Gesamt & 1 & 16 & 13 \\
\hline
\end{tabular}

\section{Größe der staatlichen Streitkräfte}

Je größer ihr Umfang, desto eher können die staatlichen Streitkräfte das gesamte Territorium kontrollieren. RebellInnen fällt es dann schwerer, einen bewaffneten Kampf gegen die Regierung zu beginnen und weiterzuführen. Zudem kann der Staat mit umfangreichen Truppen vielen VeteranInnen einen Arbeitsplatz geben, sodass sie sich nicht gegen den Frieden stellen. Trifft all dies zu, müsste mit der Größe der staatlichen Streitkräfte die Aussicht auf dauerhaften Frieden steigen. Nach einem umfangreichen Sensitivitätstest gehen Håvard Hegre und Nicholas Sambanis (2006: 526, 528) von einem robusten Zusammenhang zwischen einer großen staatlichen Streitmacht und einer geringeren Wahrscheinlichkeit eines Bürgerkrieges aus (siehe davon abweichend Bussmann 2009: 274). Arbeiten zum Rückfall in den Bürgerkrieg fügen sich zumindest teilweise in dieses Bild. Keine Studie stellt eine vergleichsweise umfangreiche Regierungsstreitmacht als statistisch signifikant rückfallfördernd heraus. Zwei Studien attestieren ihr im Gegenteil eine statistisch signifikante rückfallhemmende Wirkung (Fortna 2004: 285; Quinn et al. 2007: 186). David Mason und seine Ko-Autoren (2011: 184) erachten diesen Faktor in zwei ihrer Modelle als friedensstabilisierend, in drei anderen Modellen aber verliert der Effekt die statistische Signifikanz. In sechs Studien erreicht der Umfang der staatlichen Streitkräfte keine statistische Signifikanz. ${ }^{38}$

Tabelle 14: Große staatliche Streitkräfte sind...

\begin{tabular}{|l|c|c|c|}
\hline & $\begin{array}{c}\text { stat. signifikant } \\
\text { rückfallfördernd }\end{array}$ & $\begin{array}{c}\text { stat. signifikant } \\
\text { rückfallhemmend }\end{array}$ & $\begin{array}{c}\text { ohne stat. signifi- } \\
\text { kante Wirkung }\end{array}$ \\
\hline Studien & 0 & 2 & 6 \\
\hline Studien mit eingeschränkter Geltung & 0 & 1 & 1 \\
\hline Gesamt & 0 & 3 & 7 \\
\hline
\end{tabular}

38 Chapman/Roeder (2007: 685); Fortna (2003: 110; 2008: 106, 110); Mukherjee (2006 a: 422-424; 2006 b: 498); Sambanis (2000: 466-467, 471). 
Legt man diese Zahlen zugrunde, scheinen im Allgemeinen umfangreiche staatliche Streitkräfte dem Frieden nicht zu schaden. Ich möchte daraus aber kein Plädoyer für eine möglichst große Armee ableiten, denn mit dieser wüchse auch der Personalbedarf der Friedenstruppen, und deren konstruktive Wirkung hat die bisherige Forschung besser belegt.

\section{Wirtschaftliche Faktoren}

Wie beeinflussen das Bruttoinlandsprodukt pro Kopf und eine hohe Abhängigkeit vom Export natürlicher Ressourcen die Chancen auf stabilen Frieden?

\section{Bruttoinlandsprodukt pro Kopf}

In der Forschung zu Bürgerkriegsursachen herrscht Konsens darüber, dass ein innerstaatlicher Krieg umso unwahrscheinlicher ist, je höher das Bruttoinlandsprodukt (BIP) pro Kopf ist. Dann stehen mehr Ressourcen bereit, auf die Anliegen konkurrierender Gruppen einzugehen und das Staatsgebiet zu kontrollieren. Zudem liegen bei besseren ökonomischen Verhältnissen die Opportunitätskosten für die Teilnahme an einer Rebellion höher. ${ }^{39}$ Studien zu erneuten Bürgerkriegen weisen zwar in die gleiche Richtung, ${ }^{40}$ allerdings kommt ein erheblicher Teil von ihnen zu keinem statistisch signifikanten Ergebnis, ${ }^{41}$ womöglich mangels zuverlässiger Daten zu vielen Nachbürgerkriegsgesellschaften. ${ }^{42}$

Tabelle 15: Ein höheres BIP pro Kopf ist...

\begin{tabular}{|l|c|c|c|}
\hline & $\begin{array}{c}\text { stat. signifikant } \\
\text { rückfallfördernd }\end{array}$ & $\begin{array}{c}\text { stat. signifikant } \\
\text { rückfallhemmend }\end{array}$ & $\begin{array}{c}\text { ohne stat. signifi- } \\
\text { kante Wirkung }\end{array}$ \\
\hline Studien & 0 & 8 & 5 \\
\hline Studien mit eingeschränkter Geltung & 0 & 1 & 1 \\
\hline Gesamt & 0 & 9 & 6 \\
\hline
\end{tabular}

39 Siehe z.B. Cederman et al. (2011: 487); Wimmer et al. (2009: 329).

40 Collier (2000: 6); Collier et al. (2008: 469); Fortna (2004: 285); Glassmyer/Sambanis (2008: 372-374); Hartzell (2009: 359); Mukherjee (2006a: 422-424; 2006b: 498); Quinn et al. (2007: 186). Doyle/Sambanis (2006: 106) finden ebenso eine positive Wirkung eines höheren BIP pro Kopf, allerdings beschränkt sich ihre abhängige Variable nicht auf die Abwesenheit eines weiteren Krieges.

41 Chapman/Roeder (2007: 685); DeRouen et al. (2009: 382); Gurses et al. (2008: 147); Meernik et al. (2010: 321); Sambanis (2000: 466-467, 471). Ähnliches gilt für Sambanis/Schulhofer-Wohl (2009:108), die jedoch auf das BIP pro Kopf vor dem Bürgerkrieg schauen.

42 Aus diesem Grund erheben Hartzell/Hoddie (2007: 73) die Wirtschaftskraft nicht über diesen Indikator, sondern über die durchschnittliche Lebenserwartung. 


\section{Abhängigkeit vom Export natürlicher Ressourcen}

Blutdiamanten sind ein bekanntes Beispiel dafür, wie natürliche Ressourcen die Aussicht auf Frieden trüben. Michael L. Ross (2004: 40-42, 56-57) zählt mehrere Pfade, auf denen sie zum Bürgerkrieg führen. So können sich Rebellen die nötigen Mittel zum Kampf verschaffen, indem sie die Ressourcen selbst ausbeuten oder verkaufen, von den in diesem Sektor tätigen Unternehmen Geld abpressen oder Dritten Konzessionen zur Nutzung dieser Ressourcen in Aussicht stellen. Einschlägige Arbeiten schauen zumeist auf den Effekt einer hohen Abhängigkeit eines Landes vom Verkauf mineralischer oder biologischer Rohstoffe. Dabei erheben sie den Anteil des Exports dieser Ressourcen am BIP. In der Forschung zu Ursachen von Bürgerkriegen sieht eine Reihe von Studien einen linearen oder kurvilinearen $\mathrm{Zu}$ sammenhang zwischen dem Anteil des Exports von Rohstoffen (vor allem von Öl) und der Wahrscheinlichkeit eines Bürgerkrieges (z.B. Wiesehomeier et al. 2009: 458; Thies 2010: 329). Hegre/Sambanis (2006: 526, 528) fanden aber nur auf Basis der UCDP-Daten einen robusten Zusammenhang vor. In der Forschung zum Rückfall in den Bürgerkrieg kommen drei Studien zum Schluss, dass mit dem Anteil des Rohstoffexports am BIP die Wahrscheinlichkeit eines weiteren Bürgerkrieges in signifikanter Weise steigt. ${ }^{43}$ Colliers Befund (2000: 2-6) weist in eine ähnliche Richtung, allerdings sieht er keinen linearen Zusammenhang, sondern die höchste Wahrscheinlichkeit bei einem Anteil von $25-30 \% .{ }^{44}$ Keine Arbeit weist einer hohen Abhängigkeit vom Rohstoffexport eine statistisch signifikante rückfallhemmende Wirkung zu. Mukherjee (2006 a: 422-424) findet keine signifikante Wirkung, das Gleiche gilt bei Fortna (2004: 285) für die Zeit nach 1989.

Gelingt es, in Nachbürgerkriegsgesellschaften die Exportanteile anderer Wirtschaftszweige auszubauen, sinkt die Wahrscheinlichkeit weiterer innerstaatlicher Kriege. Diese ökonomische Transformation dürfte zumindest auf kurze Sicht schwieriger zu bewältigen sein, als das BIP pro Kopf zu steigern. Hier ist an die eingangs erwähnte Konfliktfalle zu erinnern: Ein Bürgerkrieg verheert die ökonomischen Potenziale, und das umso mehr, je heftiger und flächendeckender er tobt. Der Export von Rohstoffen gewinnt an Gewicht, da verarbeitete Produkte nicht mehr hergestellt oder abgesetzt werden können (Collier et al. 2003: 84).

43 Fortna (2004: 285); Glassmyer/Sambanis (2008: 372-374); Sambanis/Schulhofer-Wohl (2009: 108).

44 Doyle/Sambanis (2000: 788) ermitteln ebenfalls einen statistisch signifikanten destruktiven Effekt der Abhängigkeit vom Rohstoffexport, beschränken die abhängige Variable aber nicht auf die Abwesenheit des innerstaatlichen Krieges. 
Tabelle 16: Ein hoher Anteil des Exports natürlicher Ressourcen am BIP ist...

\begin{tabular}{|l|c|c|c|}
\hline & $\begin{array}{c}\text { stat. signifikant } \\
\text { rückfallfördernd }\end{array}$ & $\begin{array}{c}\text { stat. signifikant } \\
\text { rückfallhemmend }\end{array}$ & $\begin{array}{c}\text { ohne stat. signifi- } \\
\text { kante Wirkung }\end{array}$ \\
\hline Studien & 3 & 0 & 1 \\
\hline Studien mit eingeschränkter Geltung & 2 & 0 & 1 \\
\hline Gesamt & 5 & 0 & 2 \\
\hline
\end{tabular}

\section{Schluss: Nach der Forschung ist vor der Forschung}

Tabelle 17 fasst zusammen, wie oft der Befund einer statistisch signifikanten Wirkung eines Faktors in den einschlägigen Studien vertreten ist. Dabei geht sie bei den einzelnen Einflüssen von derjenigen Wirkungsrichtung aus, die häufiger ermittelt wurde als die andere. Der Einfachheit halber übernehme ich aus den vorherigen Tabellen die Gesamtzahlen und kürze die Aussagen über die Wirkungsrichtung.

Tabelle 17: Die Befunde zum Rückfall in den Bürgerkrieg im Überblick

\begin{tabular}{|lrrr|}
\hline \multicolumn{1}{|c}{$\begin{array}{c}\text { Befund: Statistisch signifikant rückfallhemmend } \\
\text { wirken... }\end{array}$} & $\begin{array}{c}\text { Anteil an allen } \\
\text { Studien }\end{array}$ & $\begin{array}{c}\text { Anteil an Studien mit } \\
\text { statistisch signifikan- } \\
\text { ten Befunden }\end{array}$ & $\begin{array}{c}\text { Zahl der } \\
\text { Studien }\end{array}$ \\
\hline ein hohes BIP pro Kopf & $60,0 \%$ & $100 \%$ & 15 \\
territoriale Machtteilung & $54,5 \%$ & $100 \%$ & 11 \\
die Präsenz von Friedenstruppen & $53,3 \%$ & $94,1 \%$ & 30 \\
Machtteilung allgemein & $52,4 \%$ & $91,6 \%$ & 21 \\
ein Sieg eher als ein Friedensabkommen & $46,7 \%$ & $77,8 \%$ & 15 \\
politische Machtteilung & $40,0 \%$ & $80,0 \%$ & 10 \\
militärische Machtteilung & $37,5 \%$ & $100 \%$ & 8 \\
(mehr) Demokratie & $36,3 \%$ & $80,0 \%$ & 11 \\
eine große staatliche Streitmacht & $30,0 \%$ & $100 \%$ & 10 \\
Separation und/oder Teilung & $28,6 \%$ & $66,7 \%$ & 7 \\
ein langer Bürgerkrieg & $26,9 \%$ & $70,0 \%$ & 26 \\
\hline Befund: Statistisch signifikant rückfallfördernd wirken ... & & & 7 \\
\hline ein hoher Anteil des Exports natürlicher Ressourcen & $71,4 \%$ & $100 \%$ & 7 \\
eine höhere Zahl von Kriegsopfern & $56,7 \%$ & $85,0 \%$ & 30 \\
ein Krieg Zwischen ethnisch definierten Parteien & $41,7 \%$ & $100 \%$ & 24 \\
eine größere Zahl an Konfliktparteien & $30,0 \%$ & $100 \%$ & 10 \\
eine Anokratie & $28,6 \%$ & $50,0 \%$ & 7 \\
\hline
\end{tabular}


Wie erwähnt, erlauben die Befunde zur Wahrscheinlichkeit eines Rückfalls nur allgemeine Folgerungen für den Umgang mit Bürgerkriegen und Nachbürgerkriegsgesellschaften, aber keine klaren Handlungsempfehlungen für einen bestimmten Fall. Zu den allgemeinen Schlüssen zählt, einen noch tobenden Krieg einzudämmen, nach dessen Ende Friedenstruppen zu stationieren und die Wirtschaft wieder auf die Beine zu stellen und zu diversifizieren. Folgerungen für einen bestimmten Fall ließen sich womöglich mit der Qualitative Comparative Analysis (QCA) gewinnen, die deterministische Kausalität zu ermitteln sucht, das Zusammenspiel von Faktoren betrachtet und aufdecken kann, dass unterschiedliche Konstellationen von Faktoren zum gleichen Ergebnis führen. Bei den Publikationen zu erneuten Bürgerkriegen steht die QCA völlig im Schatten quantitativer Verfahren. Das sollte nicht so bleiben, zumal Weiterentwicklungen der QCA auch Veränderungen auf der Zeitachse berücksichtigen können (siehe Hino 2009 zu time-Series QCA).

Sowohl Regressionsanalysen als auch Studien mittels QCA sind nur so gut wie die Daten, mit denen sie arbeiten. Hier gibt es noch Verbesserungsbedarf. In den oft genutzten Daten von Correlates of War zum Beispiel fällt eine Reihe problematischer Kodierungen ins Auge. Das Ende des Krieges in Bosnien-Herzegowina wird als Sieg eingestuft, obwohl das Dayton-Abkommen einen Kompromissfrieden herbeiführte (Gromes 2007: 142-158). Auch andere Kodierungen von Kriegsenden sind fragwürdig, wenn nicht gar offenkundig falsch. Die Probleme beginnen schon damit, was als Krieg gilt. Ein Südostasienexperte zeigte sich mir gegenüber sehr verwundert darüber, wie viele Kriege Correlates of War in den Philippinen zu zählen meinte. Die UrheberInnen von Datensätzen haben sich sehr verdient gemacht, aber vielleicht sollten sie stärker als bislang FallexpertInnen dazu einladen, die Daten zu überprüfen oder zu ergänzen. Fortzusetzen sind die Bemühungen, genauer hinzuschauen, zum Beispiel nicht auf die Vorgabe von Machtteilung in einem Friedensabkommen, sondern auf deren Umsetzung.

Abschließend möchte ich auf eine inhaltliche Frage hinweisen. Die vorgestellten Befunde lassen uns im Unklaren darüber, inwieweit eine Nachkriegsordnung einen Ausgleich zwischen den Konfliktparteien herstellen sollte, um den Frieden zu wahren. Auf der einen Seite steht der Befund, demnach Siege einen weiteren Krieg unwahrscheinlicher machen als Abkommen. Auf der anderen Seite ergab die Forschung, dass auch Abkommen mit Machtteilung und damit mit einem besonders weitgehenden Ausgleich zwischen den Kontrahenten den Frieden festigen. Ein Blick auf die jeweiligen Erklärungen dieser Befunde zeigt, wie tief der Widerspruch geht. Wer Siegen eine stärkere friedensfördernde Wirkung als Abkommen zuspricht, nennt dafür drei Argumente: Erstens führe ein Sieg allen Konfliktparteien das unausgeglichene Kräfteverhältnis vor Augen (Toft 2010 b: 33-34). Das wirke 
sich positiv aus, da eine Übermacht den Frieden fördere. Je mehr die Kontrahenten ein Ungleichgewicht ihrer Kräfte sähen, desto mehr entsprächen sich ihre Kalküle, wer von ihnen einen Krieg gewinnen würde, und desto unwahrscheinlicher führten sie Krieg gegeneinander (Blainey 1973: 56, 110-123; Wagner 1993: 242-246). Das zweite Argument zum friedensfördernden Effekt von Siegen hebt darauf ab, dass solche Triumphe die Übermacht der überlegenen Seite ausbauten. Der Sieger könne die staatlichen Ressourcen kontrollieren und seine Anliegen durchsetzen, während der Unterlegene durch die aufgezwungenen Friedensbedingungen geschwächt werde (Licklider 1995: 684-685; Toft 2010 b: 33-34, 41). Drittens wirkten sich Siege auf die Unterlegenen oftmals demoralisierend aus, wohingegen Friedensabkommen zumeist allen Konfliktparteien Zugeständnisse machten und damit alle Seiten im Glauben ließen, für eine gerechte Sache gekämpft zu haben. Dies motiviere sie dazu, mehr zu fordern, als der Kontrahent geben wolle, was die Nachkriegsordnung destabilisiere (Toft 2010 b: 152).

Träfen diese Erklärungen zu, müsste nach Friedensabkommen mit Machtteilung ein neuer Bürgerkrieg besonders wahrscheinlich sein, denn diese verkörpern das Gegenteil dessen, was die eben angeführten Argumente implizieren. Die Machtteilung gewährt allen Konfliktparteien Zugriff auf Ressourcen, mit denen sie die Nachkriegsordnung herausfordern und eine erneute militärische Konfrontation wagen können, so befürchten es jedenfalls KritikerInnen dieser Regelung (Chapman/ Roeder 2007: 681; Rothchild/Roeder 2005: 36-47; vgl. Brancati 2006: 652). Die Machtteilung schafft ein (recht) ausgeglichenes Kräfteverhältnis und verkörpert einen Kompromiss zwischen Konfliktparteien, die zuvor erfolglos versucht haben, ihr Ordnungskonzept dem Gegner aufzuzwingen. Ein solcher Kompromiss wirkt bei weitem nicht so demoralisierend wie eine militärische Niederlage. Trotzdem besteht nach Friedensabkommen mit Machtteilung keine erhöhte Wahrscheinlichkeit eines weiteren Bürgerkrieges.

Die Befunde und Erklärungen zu den Effekten von Siegen und Friedensabkommen mit Machtteilung sind offenbar nicht miteinander in Einklang zu bringen. Dies könnte sich durch den Nachweis ändern, dass Siege und Friedensabkommen mit Machtteilung unterschiedliche Wege zum stabilen Frieden weisen. Ob dies zutrifft, hat die bisherige Forschung nicht ausreichend geprüft. Aus der einen Sicht wirken ein Kräftegleichgewicht und Kompromisse zwischen den Konfliktparteien stets kriegstreibend, aus der anderen pauschal friedensfördernd. In beiden Perspektiven gilt der jeweils angenommene Effekt als so selbstverständlich, dass sie das Kräfteverhältnis und den Umfang der Kompromisse nicht eigens erheben. Sie unterstellen Siegen ein Ungleichgewicht der Kräfte und wenige bis keine Kompromisse, Abkommen hingegen ein ausgeglichenes Kräfteverhältnis und umfangreiche Kom- 
promisse. Die weitere Forschung sollte daher nicht die Art des Kriegsendes ins Zentrum zu stellen, sondern das Kräfteverhältnis und den Umfang der Kompromisse zwischen den Konfliktparteien. Dann könnte sich ein Zusammenhang zeigen, in dessen Licht die dargestellten Befunde nicht mehr widersprüchlich erscheinen, erst recht wenn nicht nur die Überlebensdaueranalyse, sondern auch die QCA zum Einsatz kommt. Möglicherweise sichert den Frieden zum einen ein Ungleichgewicht der Kräfte, das mit wenigen Kompromissen zwischen den Konfliktparteien einhergeht. Den Frieden wahrt zum anderen ein Gleichgewicht der Kräfte, das sich in umfangreichen Kompromissen widerspiegelt. Demgegenüber droht ein neuer Bürgerkrieg vor allem, wenn ein Ungleichgewicht auf umfangreiche Kompromisse trifft oder bei einem ausgeglichenen Kräfteverhältnis die Forderungen vor allem einer Seite realisiert werden.

\section{Literatur}

Blainey, Geoffrey 1973: The Causes of War, New York, NY.

Bleaney, Michael/Dimico, Arcangelo 2011: How Different Are the Correlates of Onset and Continuation of Civil Wars?, in: Journal of Peace Research 48: 2, 145-155.

Box-Steffensmeier, Janet M./Jones, Bradford S. 2004: Event History Modeling: A Guide for Social Scientists, Cambridge.

Brancati, Dawn 2006: Decentralization: Fueling the Fire or Dampening the Flames of Ethnic Conflict and Secessionism?, in: International Organization 60: 3, 651-685.

Buhaug, Halvard/Cederman, Lars-Erik/Røg, Jan Ketil 2008: Disaggregating Ethno-Nationalist Civil Wars: A Dyadic Test of Exclusion Theory, in: International Organization 62: 3, Sommer, 531-551.

Bussmann, Margit 2009: Peitsche oder Zuckerbrot? Staatskapazität und Bürgerkriege, in: Bussmann, Margit/Hasenclever, Andreas/Schneider, Gerald (Hrsg.): Identität, Institutionen und Ökonomie. Ursachen innenpolitischer Gewalt (PVSSonderheft 43), Wiesbaden, 258-282.

Cederman, Lars-Erik/Buhaug, Halvard/Røg, Jan Ketil 2009: Ethno-Nationalist Dyads and Civil War: A GIS-Based Analysis, in: Journal of Conflict Resolution 53: 4, 496-525.

Cederman, Lars-Erik/Weidmann, Nils B./Gleditsch, Kristian Skrede 2011: Horizontal Inequalities and Ethnonationalist Civil War: A Global Comparison, in: American Political Science Review 105: 3, 478-495. 
Chapman, Thomas/Roeder, Philip G. 2007: Partition as a Solution to Wars of Nationalism: The Importance of Institutions, in: American Political Science Review 101: 4, 677-691.

Collier, Paul 2000: Policy for Post-conflict Societies: Reducing the Risks of Renewed Conflict, in: http://www-wds.worldbank.org/external/default/WDSContentServer/WDSP/IB/2004/03/10/000265513_20040310161907/Rendered/PD F/28135.pdf; 8.8.2012.

Collier, Paul/Elliot, V.L./Hegre, Håvard/Hoeffler, Anke/Reynal-Querol, Anke/Sambanis, Nicholas 2003: Breaking the Conflict Trap: Civil War and Development Policy, Washington, DC.

Collier, Paul/Hoeffler, Anke 2002: On the Incidence of Civil War in Africa, in: Journal of Conflict Resolution 46: 1, 13-28.

Collier, Paul/Hoeffler, Anke 2004: Greed and Grievance in Civil War, in: Oxford Economic Papers 56: 4, 563-595.

Collier, Paul/Hoeffler, Anke/Rohner, Dominic 2009: Beyond Greed and Grievance: Feasibility and Civil War, in: Oxford Economic Papers 61: 1, 1-27.

Collier, Paul/Hoeffler, Anke/Sambanis, Nicholas 2005: The Collier-Hoeffler Model of Civil War Onset and the Case Study Project Research Design, in: Collier, Paul/Sambanis, Nicholas (Hrsg.): Understanding Civil War: Evidence and Analysis, Volume 2: Europe, Central Asia, and Other Regions, Washington, DC, 1-33.

Collier, Paul/Hoeffler, Anke/Söderbom, Måns 2008: Post-Conflict Risks, in: Journal of Peace Research 45: 4, 461-478.

Cronqvist, Lasse 2007: Konfigurationelle Analyse mit Multi-Value QCA als Methode der Vergleichenden Politikwissenschaft mit einem Fallbeispiel aus der Vergleichenden Parteienforschung (Erfolg Grüner Parteien in den achtziger Jahren), Marburg.

Cunningham, David E. 2010: Blocking Resolution: How External States Can Prolong Civil Wars, in: Journal of Peace Research 47: 2, 115-127.

DeRouen, Karl Jr./Lea, Jenna/Wallensteen, Peter 2009: The Duration of Civil War Peace Agreements, in: Conflict Management and Peace Science 26: 4, 367-387. Dixon, Jeffrey 2009: What Causes Civil Wars? Integrating Quantitative Research Findings, in: International Studies Review 11: 4, 707-735.

Dobbins, James/Jones, Seth G./Crane, Keith/Rathmell, Andrew/Steele, Brett/Teltschik, Richard/Timilsina, Anga 2005: The UN's Role In Nation-Building: From the Congo to Iraq, Santa Monica, CA. 
Doyle, Michael W./Sambanis, Nicholas 2000: International Peacebuilding: A Theoretical and Quantitative Analysis, in: American Political Science Review 94: 4, 779-801.

Doyle, Michael W./Sambanis, Nicholas 2006: Making War and Building Peace: United Nations Peace Operations, Princeton, NJ.

Falch, Ashild 2006: Durable Peace Following Civil War? Testing the Importance of International Security Guarantees and Institutional Settlement Arrangements, Master Thesis, University of Bergen, in: kms1.isn.ethz.ch/serviceengine/Files/ ISN/100242/ipublicationdocument_singledocument/cb8e3d6b-2334-4e18-a2 b 1-85bec075df47/en/2006_Durable_Peace_Following_Civil_War.pdf; 7.8.2012.

Fearon, James D. 2004: Separatist Wars, Partition, and World Order, in: Security Studies 13: 4, 394-415.

Fortna, Virginia Page 2003: Inside and Out: Peacekeeping and the Duration of Peace after Civil and Interstate Wars, in: International Studies Review 5: 4, 97-114.

Fortna, Virginia Page 2004: Does Peacekeeping Keep Peace? International Intervention and the Duration of Peace after Civil War, in: International Studies Quarterly 48: 2, 269-292.

Fortna, Virginia Page 2008: Does Peacekeeping Work? Shaping Belligerents' Choices after Civil War, Princeton, NJ.

Gates, Scott/Hegre, Havard/Gleditsch, Nils Petter 2001: Democracy and Civil Conflict after the Cold War, in: Berg-Schlosser, Dirk/Vetik, Raivo (Hrsg.): Perspectives on Democratic Consolidation in Central and Eastern Europe, New York, NY, 185-194.

Glassmyer, Katherine/Sambanis, Nicholas 2008: Rebel-Military Integration and Civil War Termination, in: Journal of Peace Research 45: 3, 365-384.

Gottwald, Eva 2009: Horowitz ernst nehmen: Der Einfluss von Leidensdruck auf den Ausbruch von Bürgerkriegen, in: Bussmann, Margit/Hasenclever, Andreas/ Schneider, Gerald (Hrsg.): Identität, Institutionen und Ökonomie. Ursachen innenpolitischer Gewalt (PVS-Sonderheft 43), Wiesbaden, 73-99.

Gromes, Thorsten 2007: Demokratisierung nach Bürgerkriegen. Das Beispiel Bosnien und Herzegowina, Frankfurt a.M.

Gurr, Ted Robert 2000: Peoples versus States: Minorities at Risk in the New Century, Washington, DC.

Gurses, Mehmet/Rost, Nicolas/McLeod, Patrick 2008: Mediating Civil War Settlements and the Duration of Peace, in: International Interactions 34: 2, 129-155. 
Hartzell, Caroline A. 1999: Explaining the Stability of Negotiated Settlements to Intrastate Wars, in: Journal of Conflict Resolution 43: 1, 3-22.

Hartzell, Caroline A. 2009: Settling Civil Wars: Armed Opponents' Fate and the Duration of the Peace, in: Conflict Management and Peace Science 26: 4, 347-365.

Hartzell, Caroline A./Hoddie, Matthew 2003: Institutionalizing Peace: Power Sharing and Post-Civil War Conflict Management, in: American Journal of Political Science 47: 2, 318-332.

Hartzell, Caroline A./Hoddie, Matthew 2007: Crafting Peace: Power-Sharing Institutions and the Negotiated Settlement of Civil Wars, University Park, PA.

Hartzell, Caroline A./Hoddie, Matthew/Rothchild, Donald 2001: Stabilizing the Peace After Civil War: An Investigation of Some Key Variables, in: International Organization 55: 1, 183-208.

Hegre, Håvard/Ellingsen, Tanja/Gates, Scott/Gleditsch, Nils Petter 2001: Toward a Democratic Civil Peace? Democracy, Political Change, and Civil War, 1816-1992, in: American Political Science Review 95: 1, 33-48.

Hegre, Håvard/Sambanis, Nicholas 2006: Sensitivity Analysis of Empirical Results on Civil War Onset, in: Journal of Conflict Resolution 50: 4, 508-535.

Hino, Airo 2009: Time-Series QCA. Studying Temporal Change through Boolean Analysis, in: Sociological Theory and Methods 24: 2, 274-226.

Hoddie, Matthew/Hartzell, Caroline 2003: Civil War Settlements and the Implementation of Military Power-Sharing Arrangements, in: Journal of Peace Research 40: 3, 303-320.

Hofmann, Tobias/Schaffer, Lena M. 2009: Einmal Frieden und zurück? Friedensmissionen und die wiederkehrende Eskalation innerstaatlicher Gewalt, in: Bussmann, Margit/Hasenclever, Andreas/Schneider, Gerald (Hrsg.): Identität, Institutionen und Ökonomie. Ursachen innenpolitischer Gewalt (PVS-Sonderheft 43), Wiesbaden, 307-332.

Horowitz, Michael C./Weisiger, Alex/Johnson, Carter 2009: Correspondence: The Limits to Partition, in: International Security 33: 4, 203-210.

Huntington, Samuel P. 1996: Democracy for the Long Haul, in: Journal of Democracy $7: 2,3-13$.

Jarstad, Anna K./Nilsson, Desirée 2008: From Words to Deeds: The Implementation of Power-Sharing Pacts in Peace Accords, in: Conflict Management and Peace Science 25: 3, 206-223.

Johnson, Carter 2008: Partitioning to Peace: Sovereignty, Demography, and Ethnic Civil Wars, in: International Security 32: 4, 140-170. 
Kaufmann, Chaim D. 1996: Possible and Impossible Solutions to Ethnic Civil Wars, in: International Security 20: 4, 136-175.

Kaufmann, Chaim D. 1998: When All Else Fails: Ethnic Population Transfers and Partitions in the Twentieth Century, in: International Security 23: 2, 120-156. Kreutz, Joakim 2010: How and When Armed Conflicts End: Introducing the UCDP Conflict Termination Dataset, in: Journal of Peace Research 47: 2, 243-250. Kumar, Radha 1997: Divide and Fall? Bosnia in the Annals of Partition, London. Licklider, Roy 1995: The Consequences of Negotiated Settlement in Civil Wars, 1945-1993, in: American Political Science Review 89: 3, 681-690.

Luttwak, Edward N. 1999: Give War a Chance, in: Foreign Affairs 78: 4, 36-44. Mason, T. David/Gurses, Mehmet/Brandt, Patrick T./Quinn, Jason Michael 2011: When Civil Wars Recur: Conditions for Durable Peace after Civil Wars, in: International Studies Perspectives 12: 2, 171-189.

Mattes, Michaela/Savun, Burcu 2009: Fostering Peace After Civil War: Commitment Problems and Agreement Design, in: International Studies Quarterly 53: 3, 737-759.

Meernik, James D./Nichols, Angela/King, Kimi L. 2010: The Impact of International Tribunals and Domestic Trials on Peace and Human Rights After Civil War, in: International Studies Perspectives 11: 4, 309-334.

Mukherjee, Bumba 2006 a: Does Third-Party Enforcement or Domestic Institutions Promote Enduring Peace After Civil Wars? Policy Lessons From an Empirical Test, in: Foreign Policy Analysis 2: 4, 405-430.

Mukherjee, Bumba 2006 b: Why Political Power-Sharing Agreements Lead to Enduring Peaceful Resolution of Some Civil Wars, But Not Others?, in: International Studies Quarterly 50: 2, 479-504.

Nilsson, Desirée 2008: Partial Peace: Rebel Groups Inside and Outside of Civil War Settlements, in: Journal of Peace Research 45: 4, 479-495.

Paris, Roland 2004: At War's End: Building Peace After Civil Conflict, Cambridge. Pearson, Frederic S./Olson Lounsbery, Marie/Walker, Scott/Mann, Sonja 2006:

Rethinking Models of Civil War Settlement, in: International Interactions 32: 2, 109-128.

Przeworski, Adam 1999: Minimalist Conception of Democracy: A Defense, in: Shapiro, Ian/Hacker-Cordón, Casiano (Hrsg.): Democracy's Value, Cambridge, 23-55.

Quinn, J. Michael/Mason, T. David/Gurses, Mehmet 2007: Sustaining the Peace: Determinants of Civil War Recurrence, in: International Interactions 33: 2, 167-193. 
Ragin, Charles C. 1987: The Comparative Method: Moving Beyond Qualitative and Quantitative Strategies, Berkeley, CA.

Regan, Patrick M. 2010: Interventions into Civil Wars: A Retrospective Survey with

Prospective Ideas, in: Civil Wars 12: 4, 456-476.

Regan, Patrick M./Norton, Daniel 2005: Greed, Grievance, and Mobilization in Civil Wars, in: Journal of Conflict Resolution 49: 3, 319-336.

Ross, Michael L. 2004: How Do Natural Resources Influence Civil War? Evidence from Thirteen Cases, in: International Organization 58: 1, 35-67.

Rothchild, Donald/Roeder, Philip G. 2005: Power Sharing as an Impediment to Peace and Democracy, in: dies. (Hrsg.): Sustainable Peace: Power and Democracy After Civil Wars, Ithaca, NY, 29-50.

Sambanis, Nicholas 2000: Partition as a Solution to Ethnic War. An Empirical Critique of the Theoretical Literature, in: World Politics 52: 4, 437-483.

Sambanis, Nicholas/Schulhofer-Wohl, Jonah 2009: What's in a Line? Is Partition a Solution to Civil War?, in: International Security 34: 2, 82-118.

Sarkees, Meredith Reid 2010: The COW Typology of War: Defining and Categorizing Wars (Version 4 of the Data), in: http://www.correlatesofwar.org/ COW2\%20Data/WarData_NEW/COW\%20Website\%20-\%20Typology\%20of \%20war.pdf.; 8.8.2012.

Sen, Amartya 1999: Democracy as a Universal Value, in: Journal of Democracy 10: 3, 3-17.

Spanger, Hans-Joachim/Schesterinina, Anastasia 2012: Demokratischer Frieden nach außen und innen? Der Forschungsstand zum Civil Democratic Peace, in: Spanger, Hans-Joachim (Hrsg.): Der demokratische Unfrieden. Über das spannungsreiche Verhältnis zwischen Demokratie und innerer Gewalt, Baden-Baden, 9-34.

Thies, Cameron G. 2010: Of Rulers, Rebels, and Revenue: State Capacity, Civil War Onset, and Primary Commodities, in: Journal of Peace Research 47: 3, 321-332.

Toft, Monica Duffy 2010 a: Ending Civil Wars: A Case for Rebel Victory, in: International Security 34: 4, 7-36.

Toft, Monica Duffy 2010 b: Securing the Peace: The Durable Settlement of Civil Wars, Princeton, NJ.

Vreeland, James Raymond 2008: The Effect of Political Regime on Civil War: Unpacking Anocracy, in: Journal of Conflict Resolution 52: 3, 401-425.

Wagner, Robert Harrison 1993: The Causes of Peace, in: Licklider, Roy (Hrsg.):

Stopping the Killing: How Civil Wars End, New York, NY, 235-268. 
Walter, Barbara F. 2002: Committing to Peace: The Successful Settlement of Civil Wars, Princeton, NJ.

Walter, Barbara F. 2004: Does Conflict Beget Conflict? Explaining Recurring Civil War, in: Journal of Peace Research 41: 3, 371-388.

Wiesehomeier, Nina/Schneider, Gerald/Braun, Constanze-Sophie 2009: Die Vereinbarkeit des Unvereinbaren? Wirtschaft und Identität als gemeinsame Bürgerkriegsursachen, in: Bussmann, Margit/Hasenclever, Andreas/Schneider, Gerald (Hrsg.): Identität, Institutionen und Ökonomie. Ursachen innenpolitischer Gewalt (PVS-Sonderheft 43), Wiesbaden, 445-470.

Wimmer, Andreas/Cederman, Lars-Erik/Min, Brian 2009: Ethnic Politics and Armed Conflict: A Configurational Analysis of a New Global Data Set, in: American Sociological Review 74: 2, 316-337.

Wolff, Jonas 2008: Turbulente Stabilität. Die Demokratie in Südamerika diesseits ferner Ideale, Baden-Baden.

\section{Der Autor}

Dr. Thorsten Gromes ist wissenschaftlicher Mitarbeiter der Hessischen Stiftung Friedens- und Konfliktforschung (HSFK) in Frankfurt a.M.

E-Mail: gromes@hsfk.de 